\title{
Wilson Sims Fall Risk Assessment Tool Versus Morse Fall Scale in Psychogeriatric Inpatients: a Multicentre Study
}

\author{
MMC Wong, PF Pang, CF Chan, MS Lau, WY Tse, LCW Lam, SKL Lee, J Tsoh, CTY Yan
}

\begin{abstract}
Objective: To compare predictive validity of the Wilson Sims Fall Risk Assessment Tool (WSFRAT) with that of the Morse Fall Scale (MFS) in psychogeriatric inpatients.

Methods: Psychogeriatric patients from Shatin Hospital, Tai Po Hospital, Castle Peak Hospital, and United Christian Hospital who had fall incident between April 2019 and April 2020 were identified. Their fall risks were assessed by the WSFRAT and the MFS, and their falls incidents during hospitalisation were recorded. Patients were classified as having high fall risk when their MFS score was $\geq 45$ and when their WSFRAT score was $\geq 7$. Sensitivity, specificity, and positive and negative predictive values of the two scales were calculated.

Results: We identified 183 (90 male and 93 female) psychogeriatric patients aged $\geq 65$ years who had fall incident and were assessed by both the WSFRAT and the MFS during the study period. Among the 183 patients, four sustained a fall during hospital stay, giving a prevalence of $2.19 \%$. All four patients were classified as having high risk of fall by WSFRAT, but only two of them were classified so by MFS. The sensitivity of WSFRAT was $100 \%$, which was higher than the $50 \%$ by MFS, but specificity of MFS was higher than that of WSFRAT ( $45.81 \%$ vs $54.75 \%)$.

Conclusion: WSFRAT is a better fall risk assessment scale for psychiatric inpatients than MFS, because of higher sensitivity (100\% vs 50\%). It has items specific to psychiatric patients and should replace MFS in psychiatric settings.
\end{abstract}

Key words: Accidental falls; Aged; Geriatric psychiatry; Risk assessment

Mimi MC Wong, Department of Psychiatry, United Christian Hospital, Hong Kong SAR, China

PF Pang, Department of Psychiatry, United Christian Hospital, Hong Kong SAR, China

CF Chan, Castle Peak Hospital, Hong Kong SAR, China

MS Lau, Castle Peak Hospital, Hong Kong SAR, China

WY Tse, Castle Peak Hospital, Hong Kong SAR, China

Linda Chiu-Wa Lam, Department of Psychiatry, The Chinese University of Hong Kong, Hong Kong SAR, China

Sarah KL Lee, Tai Po Hospital, Hong Kong SAR, China

Joshua Tsoh, Department of Psychiatry, Prince of Wales Hospital and Shatin

Hospital, Hong Kong SAR, China

Connie TY Yan, Department of Psychiatry, Shatin Hospital, Hong Kong SAR, China

Address for correspondence: Dr Mimi MC Wong, Department of Psychiatry, United Christian Hospital, Hong Kong SAR, China.

Email:wmc009@ha.org.hk

\section{Introduction}

Falls are common among elderly inpatients and may result in morbidity and mortality. ${ }^{1}$ Psychiatric inpatients are at greater fall risk owing to psychotropic medications, over-activity, and confusion. ${ }^{2,3}$ The incidence of falls in psychiatric units is higher than that of general acute care hospital units, with estimated rates of 13.1 to 25 versus 3 to 5 per 1000 inpatient days. ${ }^{2}$ In two psychiatric units in Western Australia, 139 falls were reported in 12 months; the number of falls per 1000 bed days in the two units was higher than that of any other clinical specialty units. ${ }^{4}$ Older people in psychiatric units may be restless and agitated and spend less time on their beds, unlike those in the medical ward. They may experience dizziness and is at risk of falling when getting up from chairs or beds and in bathrooms, owing to old age and concomitant comorbidities. ${ }^{2}$

Fall risk assessment tools for other hospital units may not be validated in psychiatric settings. ${ }^{5}$ The Morse Fall Scale (MFS) is intended for use in acute medical-surgical units but has not been validated in psychiatric inpatients. Toileting is a contributing factor of falling, but the MFS does not capture it. ${ }^{6}$ Although the fall risk protocol was followed in a geriatric psychiatric facility using MFS, the number of falls per 1000 patient days remained at 4.83 , which was higher than that in other hospital units. ${ }^{7}$ Psychiatric inpatients have unique risk factors for fall such as ambulatory nature, medications, anxiety, and agitation. Therefore, a falls risk assessment targeting inpatient psychiatric patients is justified. The Wilson Sims Fall Risk Assessment Tool (WSFRAT) and the Edmonson Psychiatric Fall Risk Assessment Tool are recommended for psychiatric adult and geriatric patients. ${ }^{8} 9$ One major difference between the two is the inclusion of nurse judgment in the WSFRAT. Clinical judgment by an experienced healthcare professional is as good as a fall prediction tool. ${ }^{10}$ This study 
aims to compare the sensitivity, specificity, and positive and negative predictive values of the WSFRAT and the MFS for psychogeriatric inpatients.

\section{Methods}

With reference to a study that compared MFS and the Edmonson Psychiatric Fall Risk Assessment Tool and involved 43 falls in 138 psychiatric inpatients, ${ }^{8}$ and a study of sensitivity of MFS and WSFRAT that had the prevalence of fall of around 5\%, we aimed to include 150 to 200 patients from the psychogeriatric wards of Shatin Hospital, Tai Po Hospital, Castle Peak Hospital, and United Christian Hospital. Patients who had fall incident between April 2019 and April 2020 were identified. Their fall risks were assessed by the WSFRAT and the MFS, and their falls incidents during hospitalisation were recorded. Patients were classified as having high fall risk when their MFS score was $\geq 45$ and when their WSFRAT score was $\geq 7$. Fall preventive measures were provided according to the MFS score rather than the WSFRAT score. The performance of MFS and WSFRAT in predicting the likelihood of falling was determined using a $2 \times 2$ table. The predictive validity of each tool was estimated by sensitivity, specificity, and positive and negative predictive values, with $95 \%$ confidence intervals reported. Sensitivity is calculated by the number of patients with high-risk scores who fell divided by the total number of patients who fell. Specificity is calculated by the number of patients with low-risk scores who did not fall divided by the total number of patients who did not fall. Positive predictive value is calculated by the number of patients with high-risk scores who fell divided by the total number of patients with high-risk scores. Negative predictive value is calculated by the number of patients with low-risk scores who did not fall divided by the total number of patients with low-risk scores.

\section{Results}

We identified 183 (90 male and 93 female) psychogeriatric patients aged $\geq 65$ years from Shatin Hospital $(n=30)$, Tai Po Hospital $(n=30)$, Castle Peak Hospital $(n=80)$, and United Christian Hospital $(n=43)$. They had fall incident and were assessed by the WSFRAT and the MFS between April 2019 and April 2020. We aimed to include all acute admissions so that the sample would be representative of inpatients in a psychogeriatric ward. Patients were admitted for different psychiatric conditions including behavioural and psychological symptoms of dementia, psychotic disorders, mood disorders, and alcohol or substance-related conditions. Most patients were assessed on day 1 or 2 after admission. Among the 183 patients, four sustained a fall during hospital stay, giving a prevalence of $2.19 \%$ $(95 \%$ confidence interval $=0.60 \%-5.5 \%)$. All four patients were classified as having high risk of fall by WSFRAT (score $\geq 7$ ), but only two of them were classified so by MFS (score $\geq 45$ ) [Table 1]. The sensitivity of WSFRAT was $100 \%$, which was higher than the $50 \%$ by MFS, but specificity of MFS was higher than that of WSFRAT (45.81\% vs $54.75 \%$, Table 2).

Table 1. Predictability of the Morse Fall Scale (MFS) and the Wilson Sims Fall Risk Assessment Tool (WSFRAT)

\begin{tabular}{|lccccc|}
\hline Scale & \multicolumn{5}{c|}{ No. of patients } \\
\cline { 2 - 6 } & True positive & False positive & True negative & False negative & Total \\
MFS & 2 & 81 & 98 & 2 & 183 \\
\hline WSFRAT & 4 & 97 & 82 & 0 & 183 \\
\hline
\end{tabular}

Table 2. Sensitivity, specificity, and positive and negative predictive values of the Morse Fall Scale (MFS) and the Wilson Sims Fall Risk Assessment Tool (WSFRAT)

\begin{tabular}{|lcc|}
\hline Predictive validity & \% (95\% confidence interval) \\
\cline { 2 - 3 } Sensitivity & MFS & WSFRAT \\
\hline Specificity & $50.00(6.76-93.24)$ & $100(39.76-100)$ \\
\hline Positive predictive value & $54.75(47.15-62.19)$ & $45.81(38.36-53.41)$ \\
\hline Negative predictive value & $2.41(0.91-6.25)$ & $3.96(3.48-4.51)$ \\
\hline
\end{tabular}




\section{Discussion}

The clinical implication of low sensitivity is that a substantial number of patients who are at high risk for falling will not receive fall prevention intervention measures. Thus, sensitivity is a more important measure of the efficacy of the fall-risk assessment tool, ${ }^{11}$ as it is used to identify patients at high risk so that timely intervention can be implemented to prevent falls.

Both the WSFRAT and the MFS assess risk factors such as history of fall, mental status, physical status, gait, and ambulatory aids needed. The main differences between the two scales are that WSFRAT has items about elimination issues and psychiatric medications, whereas MFS has items for intravenous access, which is unusual for psychiatric inpatients. In the present study, psychogeriatric patients who were classified as having high fall risk by WSFRAT but not by MFS had considerable scoring contributed by medications and/or elimination problems. This also applied to the two patients who fell but had been classified by MFS as having low fall risk. Psychogeriatric inpatients frequently fall on the way to the bathroom..$^{12}$ Patients deemed to have fall risk are advised to seek help before leaving their bed or chair, but they often go to the bathroom without calling for assistance, especially those with cognitive impairment. WSFRAT includes elimination issues in the scoring and those who are independent but have urinary urgency or frequency are scored higher in fall risk. These patients may take risk to avoid soiling themselves.

Clinical judgment of nurses is important when using the WSFRAT. Nurses can upgrade the fall risk from low to high when clinically indicated. This compensates for any factors that are not included or reflected in the scoring but may increase the risk of fall. Reasons for upgrading fall risk include recent history of fall, repeated falls, and lack of safety awareness. In the present study, only eight patients were upgraded by nurses. The small number may be due to the comprehensive psychiatric assessment by the WSFRAT that had already included most patients with fall risk. Nonetheless, when using MFS, it was not uncommon for nurses to upgrade the rating as well. This reflects a need for clinical judgment, but there is no official instruction by MFS to facilitate the upgrade, and some frontline staff may be uncomfortable to adjust the scoring.

Psychogeriatric inpatients are commonly put on multiple psychiatric medications. The likelihood of falling increases with the use of neuroleptic and antipsychotic drugs. ${ }^{13}$ Combination of psychotropics or other drugs may increase the sedating effect and drug interactions. Fall risk is associated with polypharmacy when at least one fall riskincreasing drug is part of the daily regimen..${ }^{14}$ Patients are scored higher in WSFRAT when taking more psychotropics and when starting a new drug. Those having detoxification are regarded as having high fall risk, as high dosage of benzodiazepine may be used.

Both scales are easy to use and quick to complete. Nurses commented that the items of WSFRAT were relevant to psychiatric patients and that clinical judgment of nurses was included. WSFRAT identified patients at high risk for falling who were considered low risk by MFS and facilitated implementation of fall prevention interventions by nurses.

There are limitations to the present study. The prevalence of fall was lower than expected. This was what we aimed for. The psychiatric inpatients were assessed by the two fall assessment scales, and additional fall prevention measures might have been implemented and resulted in fewer falls. The WSFRAT was an extra assessment on top of the routine MFS assessment. It was performed only once upon admission by busy staff. In addition, the interrater reliability was not evaluated. It is unknown whether data collectors on each unit agreed with each other's assessments, although briefing sessions were conducted in all four centres to align the practice and scoring before the study. Furthermore, the sample size and the number of falls were small. Detailed background information of patients and the duration of hospital stay were not obtained.

\section{Conclusion}

WSFRAT is a better fall risk assessment scale for psychiatric inpatients than MFS, because of higher sensitivity (100\% vs $50 \%$ ). It has items specific to psychiatric patients and should replace MFS in psychiatric settings.

\section{Contributors}

All authors designed the study and acquired the data. MMC Wong analysed the data and drafted the manuscript. All authors critically revised the manuscript for important intellectual content. All authors had full access to the data, contributed to the study, approved the final version for publication, and take responsibility for its accuracy and integrity.

\section{Conflicts of interest}

All authors have disclosed no conflicts of interest.

\section{Funding/support}

This study received no specific grant from any funding agency in the public, commercial, or not-for-profit sectors.

\section{Data Availability}

All data generated or analysed during the present study are available from the corresponding author on reasonable request.

\section{Ethics approval}

The study was approved by the New Territories West Cluster Research Ethics Committee, the Joint Chinese University of 
Hong Kong-New Territories East Cluster Clinical Research Ethics Committee, and the Kowloon Central/Kowloon East Research Ethics Committee (Ref: CRE-2018.632, CRE2019.272, NTWC/REC/18105 and REC (KC/KE)-18-0258/ ER-4).

\section{Acknowledgements}

The study has been published in the Hospital Authority Quality and Safety Annual Report 2020. We thank Ms Catherine Cheng, Mr Kin Hang Wong, Mr Samuel Kong, Mr HW Leung, Ms Kit Ping Ho, Mr Raymond Wai Man Cheuk, Ms Elsie Wong, Ms Wing Chong Ngai, Ms Sin Yi Lee, Ms Pik Yan Lo, Mr Wai Lok Lai, and Mr Chi Kin Leung of the psychogeriatric wards of Castle Peak Hospital, Shatin Hospital, Tai Po Hospital, and United Christian Hospital for assistance in data collection.

\section{References}

1. Oliver D, Britton M, Seed P, Martin FC, Hopper AH. Development and evaluation of evidence based assessment tool (STRATIFY) to predict which elderly inpatients will fall: case-control and cohort studies. BMJ 1997;315:1049-53. Crossref

2. Blair E, Gruman C. Fall in an inpatient geriatric psychiatric population. J Am Psychiatr Nurses Assoc 2005;11:351-4. Crossref

3. Finkelstein E, Prabhu M, Chen H. Increased prevalence of falls among elderly individuals with mental health and substance abuse conditions.
Am J Geriatr Psychiatry 2007;15:611-9. Crossref

4. Heslop K, Wynaden D, Bramanis K, Connolly C, Gee T, Griffiths R, et al.Assessing falls risk in older adult mental health patients: a Western Australian review. Int J Ment Health Nurs 2012;21:567-75. Crossref

5. Rutledge D, Donaldson N, Pravikoff D. Fall risk assessment and prevention in hospitalized patients. OJCI 2003;6:1-55.

6. Tzeng HM. Understanding the prevalence of inpatient falls associated with toileting in adult acute care settings. J Nurs Care Qual 2010;25:2230. Crossref

7. Knight M, Coakley C. Fall risk in patients with acute psychosis. J Nurs Care Qual 2010;25:208-15. Crossref

8. Edmonson D, Robinson S, Hughes L. Development of the Edmonson Psychiatric Fall Risk Assessment Tool. J Psychosoc Nurs Ment Health Serv 2011;49:29-36. Crossref

9. Abraham SP. Looking for a psychiatric fall risk assessment tool? Ann Psychiatry Ment Health 2016;4:1061.

10. Wilson SC, Fettes S, Sims K. Gravity: It's not a suggestion-It's the law! The development of the Wilson Sims Psychiatric Fall Risk Assessment. J Am Psychiatr Nurses Assoc 2014;20:83.

11. Vassallo M, Stockdale R, Sharma JC, Briggs R, Allen S. A comparative study of the use of four fall risk assessment tools on acute medical wards. J Am Geriatr Soc 2005;53:1034-8. Crossref

12. Wong MM, Pang PF. Factors associated with falls in psychogeriatric inpatients and comparison of two fall risk assessment tools. East Asian Arch Psychiatry 2019;29:10-4. Crossref

13. Woolcott JC, Richardson KJ, Wiens MO, Patel B, Marin J, Khan KM, et al. Meta-analysis of the impact of 9 medication classes on falls in elderly persons. Arch Intern Med 2009;169:1952-60. Crossref

14. Ziere G, Dieleman JP, Hofman A, Pols HA, van der Cammen TJ, Stricker BH. Polypharmacy and falls in the middle age and elderly population. Br J Clin Pharmacol 2006;61:218-23. Crossref 